\title{
Research on Key Technologies of Bandwidth Optimizations for Ad Hoc Networks
}

\author{
Hua Zhang \\ Guangzhou Institute of Railway Technology, Guangzhou, Guangdong, 510430, China
}

\begin{abstract}
The bottleneck in the development of ad hoc network is analyzed. Points out the physical layer application of new transmission technology is network bandwidth optimization approach, however, strengthen the its effectiveness and performance needs reasonable design of MAC layer and routing layer and cross layer design, explains some kind of transmission technology of MAC layer and routing layer characteristics and challenges faced.
\end{abstract}

KEYWORD: Optimization; Wireless transmission; Tactical ad hoc networks; Routing protocol.

\section{INTRODUCTION}

Hoc Ad network is a mobile communication network formed by a group of autonomous wireless nodes. Due to the ad hoc network can be widely used in military wireless communication, mobile conference, home networking, emergency services, sensor networks, personal area network and other commercial applications, military actual and potential application prospect makes it's become the basis of ad hoc network. Therefore, the research on ad hoc networks especially ad hoc network has very important significance (Xu Li, 2012). Along with the improvement of military modernization and the degree of information, the demand for communication ability becomes more and more high, the contradiction between the bandwidth and the demand is inevitable. Therefore, for the hoc Ad network bandwidth supply will not meet its needs, the need to build a dedicated bandwidth technology research.

At present, although there are many research on the optimization of bandwidth and improve the spectrum utilization, but it is not clear that the hoc Ad network bandwidth optimization technology related research results. This paper points out the key technologies of hoc Ad network bandwidth optimization, in order to achieve a significant improvement in the physical layer spectrum utilization and efficient utilization of wireless network resources. The establishment of a Hoc Ad network based on the wireless resource allocation mechanism and planning theory framework, the new bandwidth expansion of radio resource allocation mode.

\section{KEY TECHNOLOGIES IN WIRELESS MOBILE TRANSMISSION}

In order to meet the new application of hoc Ad network and higher communication needs, more advanced wireless transmission technology is needed. The physical layer transmission technology which can provide greater bandwidth are emerging in endlessly, which brings new opportunities for the further development of hoc Ad network.

Smart antenna technology to improve spatial reuse capability 。 Smart antenna is one of the key technologies of mobile communication in the future. Directional antennas can be from the space separating signal, effectively reduce the access protocol for multiple access interference, improve channel spatial reuse, making the same time were communication of the increase in the number of nodes, thus increasing network capacity. The gain of directional antenna in a particular direction increased, thereby increasing the transmission distance of the node, increase the network connectivity. For multi hop Ad Hoc network, reduces the packet forwarding hops, save network overhead. Directional antennas can form a narrow beam in the desired direction, and the gain in the other direction is very small, so as to effectively enhance the anti-interference capability of signal. Therefore, directional antenna has a unique advantage to enhance the anti-interference capability of the network in the tactical environment. When a directional antenna is used as a receiver, the main 
beam can be aligned with the useful signal direction, and the zero alignment interference signal is used to filter the interference signal in the space. The high anti-interference ability of directional antenna not only enhances the reliability of the network, but also increases the capacity of the network.

\section{AD HOC NETWORK BASED ON NEW TRANSMISSION TECHNOLOGY}

The new transmission technology provides high spatial multiplexing capability, high transmission capacity, high anti-interference ability to meet the requirements of future hoc Ad networks for these aspects of performance. Therefore, it is very necessary to adopt new transmission technology in hoc Ad network, especially for military hoc Ad networks. Because the MAC protocol is the core part of the Ad Hoc network. In the Ad hoc network transmission technology application conditions under the new MAC protocol and the existing routing protocols can not fully play its role, can not reflect the superiority of the new technology brings. In order to speed up the reference of new technology, give full play to its remarkable characteristics, must be modified or re design of existing protocols.

\subsection{Multi access of hoc Ad network based on new technology}

The application of new wireless transmission technology can effectively improve the system performance and system capacity. If the MAC layer is independent of the physical characteristics, and only takes into account the "worst case" allocation of radio resources, it will reduce the effectiveness of the protocol and waste system resources. Re design the MAC protocol, not only solve the technical problems in the prior art such as hidden terminal and exposed terminal problems, fair problem, QoS guarantee, should also taking into account the specific characteristics of physical layer and adaptive to fit business needs to more efficient use of system resources

\subsection{Hoc Ad network routing based on new transmission technology.}

New transmission technology has been in the physical layer is widely studied, however, to strengthen their effectiveness and performance only through reasonable design of a high-rise, including not only the MAC layer, and the routing layer to effectively strengthen. At present, the research in this area is mostly focused on the MAC protocol, and the research on routing technology is still few.

The collaborative technology route can be divided into two categories based on respectively routing strategy based on energy and routing strategy based on bandwidth. Is a good choice a from the source node to the destination node routing based on by in each hop nodes according to the improvement of bandwidth selection to carry out coordinated, so as to improve the transmission capability of the routing. Another way is in the routing and taking into account the effect of collaborative technology of per hop transmission bandwidth, so as to determine the per hop transmission is the cooperative communication technology, and select the maximum bandwidth routing for data transmission.

\subsection{Cross layer design}

Layered architecture, such as the seven layer open system interconnection model, defines the level of service provided by each layer. In each layer of the business is achieved through the design protocol for each layer, it can be achieved in the target platform to get a complete system. In the design phase, designers have two options. Protocol can be designed by following the rules of the original architecture. In a hierarchical OSI reference model, this may mean that the design protocol is simply the use of a low level of service, and there is no need to care about the details of how these services are provided. This also means that the protocol does not require any new interfaces in the reference architecture. In addition, the protocol can be designed by violating the original architecture. To meet the specific requirements of ad hoc networks, it is necessary to analyze the protocols at various layers of mutual influence, mutual restriction relation, put forward a at multiple levels of the protocol stack support adaptive and implementation performance priority and cross layer architecture.

The hypothetical reference architecture has application layer, transport layer, network layer, link layer (it includes data link control (DLC) and media access control (MAC) sub layer) and physical layer, all of these layers complete their function. Cross layer feedback means that each layer in the protocol stack can interact with other layers. A layer can not only interact with the upper layer, but also with the lower layer. The layer of information interaction includes application layer, transport layer, network layer, link layer and physical layer. Each layer can interact with other layers of the parameters and its interaction mechanism is described.

When the new wireless transmission technology, physical layer characteristics to the upper layer protocol design effect is larger, such as antenna gain, power, beam characteristics are on the performance of MAC and routing protocols produced great influence, be considered jointly. The MAC protocol and routing protocol have the common information, such as neighbor information, antenna pointing and so on. Moreover, the QoS guarantee needs the comprehensive consideration of MAC and routing protocols. Therefore, the protocol can be used in this layer to 
respond to changes in other layers. If the regulation mechanism of this layer can not be competent, then the upper level of performance indicators will also be affected, and will call the upper layer of the adaptive mechanism to overcome or reduce the performance of the lower layer can not solve the problem. If the MAC system is independent of the physical layer characteristics, and only take into account the "worst case", it will be a waste of system resources in MIMO.

Cross layer design will span several areas of communication, network, system structure and Realization of closely linked together, there is no consistent solution, and there are still a number of open problems, which some problems only from the view of the performance of can not be solved, the need to consider the system structure. The potential performance gain always urges the designers to do the cross layer design without following the layered architecture. Cross layer design in the design stage it shows that the protocol architecture of hierarchical violation. Therefore it is natural to ask a question, "will there be a general architecture for wireless network protocol design, and do not violate them?". In fact, this is a difficult and is worthy of further study.

\section{SUMMARY}

With the increasing demand of the battlefield, the application of the new wireless transmission technology is an inevitable trend of the development of hoc Ad network. Bandwidth is a need to manage the limited resources and traditional bandwidth problem confined to the physical layer transmission channel analysis. In this paper, the bandwidth optimization problem study is extended to the MAC layer bandwidth control and cross layer bandwidth management category, increasing the choice of ways to solve the problem of bandwidth, improve the utilization of bandwidth resources. Using new technology to increase the capacity of a single link, such as multiple input multiple output (MIMO) antenna, to the antenna, the use of higher frequency bands. Integrated with a variety of bandwidth and sharing, strengthen and expand channel band width based on can maximally improve network capacity, can solve the ad hoc network information "sudden" change and information blocking problem. New technology will greatly improve the capacity, but the unrestricted user needs will likely be synchronized or exceeded the obtained capacity, but not a single technology can solve this problem. How to put forward more practical application of networking protocols in the new wireless transmission network is an important direction of hoc Ad network technology research in the future.

\section{REFERENCES}

M. Ali, B.G Stewart,A Shahrabi,etc. Congestion Adaptive Multipath Routing For Load Balancing in Mobile Ad hoc Networks[J], Journal of Computer Science,2013,8(5),305309.

Ni S, Tseng Y, Chen Y, Sheu J. The Broadcast Storm Problem in a Mobile Ad hoc Network, International Conference on Mobile Computing and Networking, 1999[C]. Seattle, USA: IEEE Communications Society, 1999: 151-162.

Xu Li,Amiya Nayak,Ivan Stojmenovic. Achieving Load Awareness in Position-based Wireless Ad Hoc Routing[J], Journal of Covergence, 2012,3 (3),17-21. 